\title{
Collecting a Large Scale Dataset for Classifying Fake News Tweets Using Weak Supervision
}

\author{
Stefan Helmstetter and Heiko Paulheim *(D)
}

Citation: Helmstetter, S.; Paulheim H. Collecting a Large Scale Dataset for Classifying Fake News Tweets Using Weak Supervision. Future Internet 2021, 13, 114. https:// doi.org/10.3390/fi13050114

Academic Editor: Jari Jussila

Received: 23 March 2021

Accepted: 26 April 2021

Published: 29 April 2021

Publisher's Note: MDPI stays neutral with regard to jurisdictional claims in published maps and institutional affiliations.

Copyright: (c) 2021 by the authors. Licensee MDPI, Basel, Switzerland. This article is an open access article distributed under the terms and conditions of the Creative Commons Attribution (CC BY) license (https:/ / creativecommons.org/licenses/by/ $4.0 /)$.
Data and Web Science Group, School of Business Informatics and Mathematics, University of Mannheim, B6 26, 68159 Mannheim, Germany; stefanhelmstetter@web.de

* Correspondence: heiko@informatik.uni-mannheim.de

\begin{abstract}
The problem of automatic detection of fake news in social media, e.g., on Twitter, has recently drawn some attention. Although, from a technical perspective, it can be regarded as a straight-forward, binary classification problem, the major challenge is the collection of large enough training corpora, since manual annotation of tweets as fake or non-fake news is an expensive and tedious endeavor, and recent approaches utilizing distributional semantics require large training corpora. In this paper, we introduce an alternative approach for creating a large-scale dataset for tweet classification with minimal user intervention. The approach relies on weak supervision and automatically collects a large-scale, but very noisy, training dataset comprising hundreds of thousands of tweets. As a weak supervision signal, we label tweets by their source, i.e., trustworthy or untrustworthy source, and train a classifier on this dataset. We then use that classifier for a different classification target, i.e., the classification of fake and non-fake tweets. Although the labels are not accurate according to the new classification target (not all tweets by an untrustworthy source need to be fake news, and vice versa), we show that despite this unclean, inaccurate dataset, the results are comparable to those achieved using a manually labeled set of tweets. Moreover, we show that the combination of the large-scale noisy dataset with a human labeled one yields more advantageous results than either of the two alone.
\end{abstract}

Keywords: fake news; Twitter; weak supervision; source trustworthiness; social media

\section{Introduction}

In recent years, fake news shared on social media has become a much recognized topic [1-3]. Social media make it easy to share and spread fake news, i.e., misleading or wrong information, easily reaching a large audience. For example, during the 2016 presidential election in the United States, recent research revealed that during the election campaign, about 14 percent of Americans used social media as their major news source, which dominates print and radio [4].

The same research work found that false news about the two presidential candidates, Donald Trump and Hillary Clinton, were shared millions of times in social media. Likewise, in the 2021 US presidential election campaign, recent research works discovered larger misinformation campaigns around the topic of COVID-19 [5]. Moreover, in the aftermath of the 2021 election, fake news campaigns claiming election fraud were detected [6]. These examples show that methods for identifying fake news are a relevant research topic.

While other problems of tweet classification, e.g., sentiment detection [7] or topic detection [8], are rather extensively researched, the problem of fake news detection, although similar from a technical perspective, is just about to gain attention.

The identification of a news tweet into fake or non-fake news is a straight forward binary classification problem. Classification of tweets has been used for different use cases, most prominently sentiment analysis [9], but also by type (e.g., news, meme, etc.) [10], or relevance for a given topic [11]. 
In all of those cases, the quality of the classification model strongly depends on the amount and quality of training data. Thus, gathering a suitable amount of training examples is the actually challenging task. While sentiment or topic can be more easily labeled, also by less experienced crowd workers [9,12], labeling a news tweet as fake or non-fake news requires a lot more research, and may be a non-trivial task. For example, web sites like Politifact (http:/ / www.politifact.com/, accessed on 31 Auguest 2017), which report fake news, employ a number of professional journalists for this task.

In this paper, we follow a different approach. Instead of aiming at a small-scale handlabeled dataset with high-quality labels, we collect a large-scale dataset with low-quality labels. More precisely, we use a different label, i.e., the trustworthiness of the source, as a noisy proxy for the actual label (the tweet being fake or non-fake) This may introduce false positives (since untrustworthy sources usually spread a mix of real and fake news), as well as occasional false negatives (false information spread by trustworthy sources, e.g., by accident), although we assume that the latter case is rather unlikely and hence negligible. We show that the scarcity of hand-labeled data can be overcome by collecting such a dataset, which can be done with very minimal labeling efforts. Moreover, by making the data selection criteria transparent (the dataset consists of all tweets from a specified set of sources over a specified time interval), we can mitigate problems of biased data collection [13].

In other words: we build a large scale training dataset for a slightly different task, i.e., predicting the trustworthiness of a tweet's source, rather than the truth of the tweet itself. Here, we follow the notion of weakly supervised learning, more specifically, learning with inaccurate supervision, as introduced by [14]. We show that a classifier trained on that dataset (which, strictly speaking, is trained for classifying tweets as coming from a trustworthy or a non-trustworthy source) can also achieve high-quality results on the task of classifying a tweet as fake or non-fake, i.e., an F1 score of up to 0.9. Moreover, we show that combining weakly supervised data with a small set of accurately labeled data brings additional advantages, e.g., in the area of constructing distributional features, which need larger training corpora.

The rest of this paper is structured as follows. In the next section, we give an overview on related work. The subsequent sections describe the dataset collection, the classification approach, and an evaluation using various datasets. We close the paper with a conclusion and an outlook on future work. A full description of the features used for the classification is listed in an appendix. A preliminary study underlying this paper was published at the IEEE/ACM International Conference on Advances in Social Networks Analysis and Mining (ASONAM) [15].

\section{Related Work}

Although fake news in social media is an up-to-date topic, not too much research has been conducted on the automatic detection of fake news. There are, however, some works which focus on a related question, i.e., assessing the credibility of tweets.

Ref. [16] analyze the credibility of tweets related to high impact events using a supervised approach with a ranking support vector machine algorithm. As a training set a sample of the collected data was manually annotated by three annotators (14 events $\times 500$ tweets) based on related articles in well-known newspapers. Events were selected through trending topics collected in a specified period. Features independently of the content of a tweet, and content specific features like unigrams as well as user features were found helpful. Hereby content specific features were as important as user features. It was found out that the extraction of credible information from Twitter is possible with high confidence.

Ref. [17] derive features for credibility based on a crowd-sourced labeling, judging, and commenting of 400 news tweets. Through association rule mining, eight features were identified that humans relate with credibility. Politics and breaking news were found to be more difficult to rate consistently. 
Ref. [18] follow a different approach to assess the credibility of tweets. Three datasets were collected, each related to an event. One was labeled on the basis of network activity, the others were manually annotated. Two different approaches were proposed and then fused in the end. On the one hand, a binary supervised machine learning classification was performed. On the other hand, an unsupervised approach using expectation maximization was chosen. In the latter, tweets were clustered into similar groups, i.e., claims. To that end, a network was built from sources connected to their claims and vice versa. The topology was then used to compute the likelihood of a claim being correct. Depending on the dataset, the methods gave quite different results. Nevertheless, the fusions improved the prediction error. To summarize, the supervised approach showed the better accuracy compared to the expectation maximization when considering even non-verifiable tweets. However, it does not predict the truthfulness of tweets.

Ref. [19] also propose a supervised approach for assessing information credibility on Twitter. A comprehensive set of message-, user-, topic-, and propagation-based features was created. The latter refer to a propagation tree created from retweets of a message. A supervised classifier was then built from a manually annotated dataset with about 600 tweets separated into two classes, one that states that a news tweet is almost certainly true, another one for the residual. A J48 decision tree performed best within a 3-fold cross validation and reached a classification accuracy of $86 \%$. Further experiments show that a subset with the propagation related features as well as a top-element subset are both very relevant for the task. Thereby, the top-element subset only includes tweets with the most frequent URL, hashtag, user mention, or author. However, the authors claim that user and tweet features only are not sufficient to assess the credibility of a tweet.

Most of these approaches share the same characteristics:

1. They use datasets that are fairly small (less than 10,000 Tweets),

2. they use datasets related to only a few events, and

3. they rely on crowd sourcing for acquiring ground truth.

The first characteristic may be problematic when using machine learning methods that require larger bodies of training data. The second and the third characteristic may make it difficult to update training datasets to new events, concept drift, shifts in language use on Twitter (e.g., possibly changes caused by switching from 140 to 280 characters), etc.

In contrast, the approach discussed in this paper acquires a dataset for the task of fake news detection requires only minimal human annotation, i.e., a few lists of trustworthy sources. Therefore, the process of acquiring the dataset can be repeated, gathering a large-scale, up-to-date dataset at any time.

As the topic of fake news detection has recently drawn some attention, there are a few approaches which attempt to solve related, yet slightly different tasks, e.g., determining fake news on Web pages [20-22]. Since those operate on a different type of content and hence can exploit a different set of features, they are not quite comparable. Ref. [23] present a descriptive study of how fake news spread on Twitter, which was able to reveal characteristic patterns in the spreading of fake and non-fake news, but was not used as a predictive model.

\section{Datasets}

We use two datasets in this work:

1. A large-scale training dataset is collected from Twitter and labeled automatically. For this dataset, we label tweets by their sources, i.e., tweets issued by accounts known to spread fake news are labeled as fake, tweets issued by accounts known as trustworthy are labeled as real.

2. A smaller dataset is collected and labeled manually. This dataset is not used for training, only for validation.

In the following, we describe the datasets and their collection in more detail. 


\subsection{Large-Scale Training Dataset}

We create our training dataset by first collecting trustworthy and untrustworthy sources. Then, for each of the sources, we collect Tweets using the Twitter API. Each tweet from a trustworthy source is labeled as real news, each tweet from an untrustworthy source is labeled as fake news.

While this labeling can be done automatically at large scale, it is far from perfect. Most untrustworthy sources spread a mix of fake and real news. The reverse (i.e., a trustworthy source spreading fake news, e.g., by accident) may also occur, but we assume that this case very rare, and hence do not consider it any further.

For collecting fake news sources, we use lists from different Web pages:

- http://mashable.com/2012/04/20/twitter-parodies-news/\#IdNx6sIG.Zqm (accessed on 24 February 2017)

- https://www.dailydot.com/layer8/fake-news-sites-list-facebook/ (accessed on 24 February 2017)

- https://www.cbsnews.com/pictures/dont-get-fooled-by-these-fake-news-sites/ (accessed on 24 February 2017)

- http://fakenewswatch.com/ (accessed on 24 February 2017)

- $\quad$ https://www.snopes.com/2016/01/14/fake-news-sites / (accessed on 24 February 2017)

- https://www.thoughtco.com/guide-to-fake-news-websites-3298824 (accessed on 24 February 2017)

- $\quad$ https://newrepublic.com/article/118013/satire-news-websites-are-cashing-gullibleoutraged-readers (accessed on 24 February 2017)

- http://www.opensources.co/ (accessed on 24 February 2017).

In total, we collected 65 sources of fake news.

For collecting trustworthy news sources, we used a copy of the recently shut down DMOZ catalog (http:/ / dmoztools.net/ (accessed on 25 February 2017), as well as those news sites listed as trustworthy in opensources, and filtered the sites to those which feature an active Twitter channel. In order to arrive at a balanced dataset, we collected 46 trustworthy news sites. That number is incidentally chosen lower than that of fake news sources, since we could collect more tweets from the trustworthy sites.

In the next step, we used the Twitter API (https://developer.twitter.com/en/docs (accessed on 26 April 2021) to retrieve tweets for the sources. The dataset was collected between February and June 2017. Since the Twitter API only returns the most recent 3200 tweets for each account (https:/ / developer.twitter.com/en/docs/tweets/timelines/ api-reference/get-statuses-user_timeline.html (accessed on 26 April 2021), the majority of tweets in our dataset is from the year 2017, e.g., for an active twitter account with 20 tweets per day, that limitation Twitter API allows us retrieve tweets for the past 160 days (The new Twitter API v2, which is being rolled out since the beginning of 2021, has removed some of those limitations: https://developer.twitter.com/en/docs/twitter-api/ early-access (accessed on 26 April 2021).

In total, we collected 401,414 examples, out of which 110,787 (27.6\%) are labeled as fake news (i.e., they come from fake news sources), while 290,627 (72.4\%) are labeled as real news (i.e., they come from trustworthy sources). Figure 1 shows the distribution of tweets by their tweet time. Due to the collection time, the maximum tweet length is 140 characters, since the extension to 280 characters was introduced after our data collection (https: / / blog.twitter.com/en_us/topics/product/2017/tweetingmadeeasier.html (accessed on 26 April 2021). Figure 2 shows the topical distribution of the tweets. Figure 3 depicts further statistics about the tweets in the training set. It can be observed that while there is no strong difference in the sentiment (average 0.39 on the real class, 0.38 on the fake class) and the subjectivity score (average 0.27 on the real class, 0.29 on the fake class), the number of retweets (average 123 on the real class, 23 on the fake class) and favorites (average 236 on the real class, 34 on the fake class) differ considerably. 


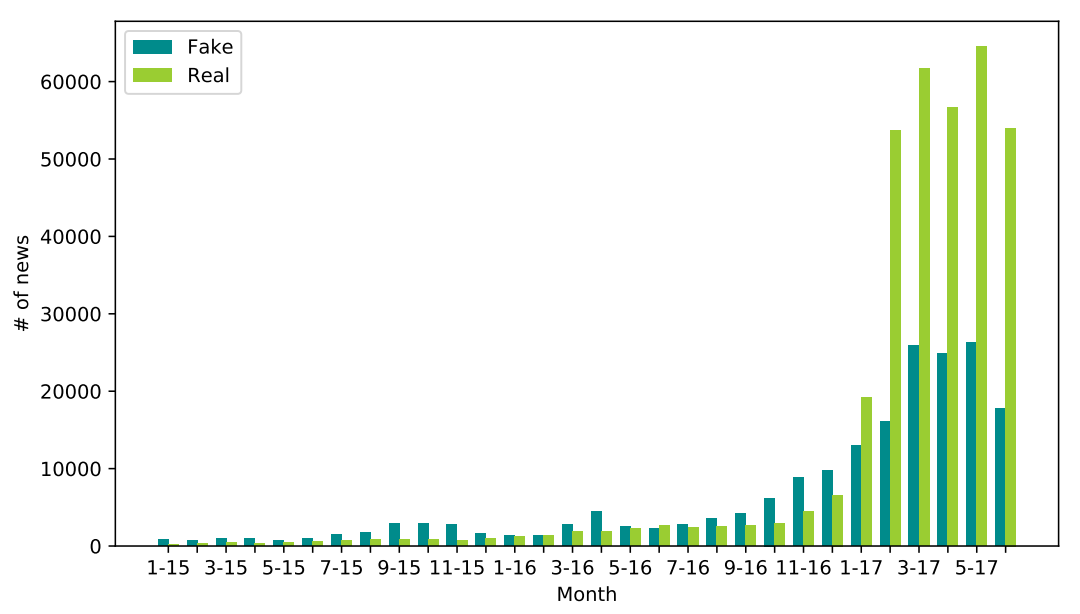

Figure 1. Distribution of tweets labeled as real and fake news in the training dataset.

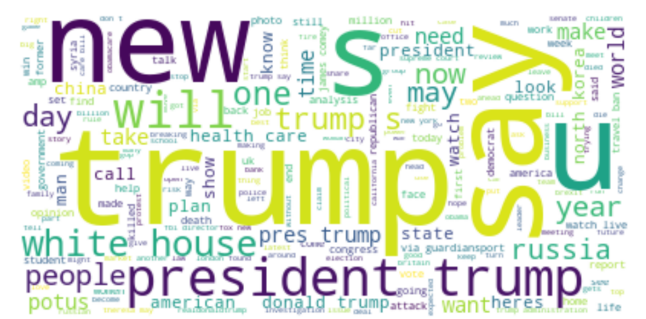

(a) Word cloud of the real news class

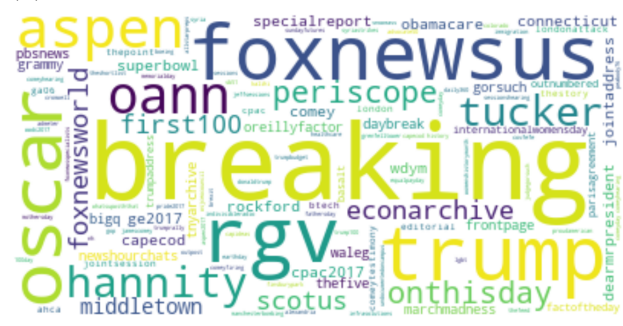

(c) Tag cloud of the real news class

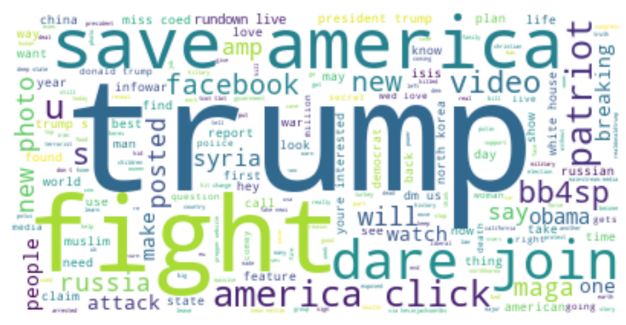

(b) Word cloud of the fake news class

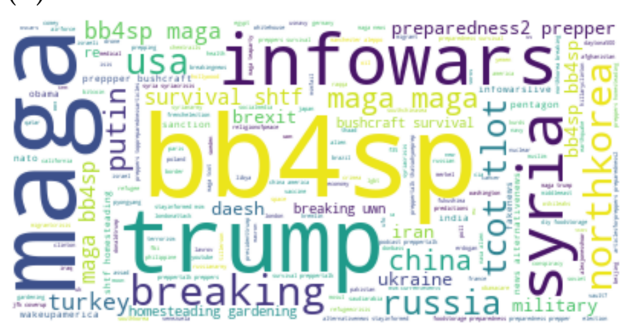

(d) Tag cloud of the fake news class

Figure 2. Topic distribution in the training dataset.

It is important to point out that for collecting more than $400 \mathrm{k}$ tweets, the actual annotation workload was only to manually identify 111 sources. In other words, from 111 human annotations (trustworthy vs. untrustworthy source), we produce $400 \mathrm{k}$ annotated tweets.

As discussed above, we expect the real news class to contain only a negligible amount of noise, but we inspected the fake news class more closely. The results are depicted in Table 1. The fake news class contains a number of actual news (a phenomenon also known as mixed information [24]), as well as tweets which are not news, but other contents (marked as "no news" in the table). These numbers show that the fake news tweets are actually the smallest class in the training sample. However, since the sample contains both real and fake news tweets from the same period of time, we can assume that for real news, those will also appear in the class labeled as non-fake, and since the real news class is larger by a factor of three, the classifier will more likely label them as real news. For example, if a real news item is tweeted by eight real and two fake news sources, a decent classifier would, to put it very simply, learn that it is a real news item with $80 \%$ confidence. This shows that the incidental imbalance of the dataset towards real news is actually useful. 


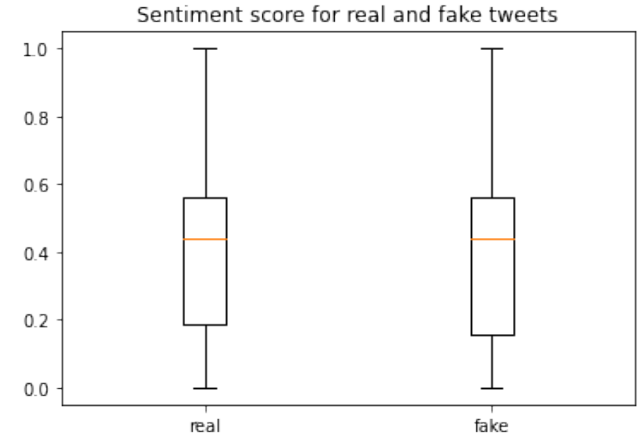

(a) Sentiment score

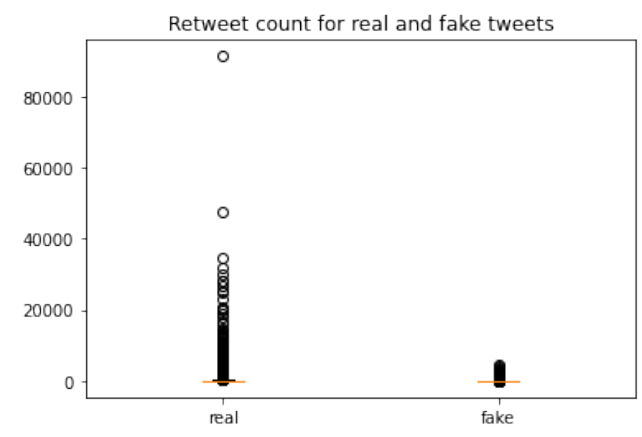

(c) Retweet count

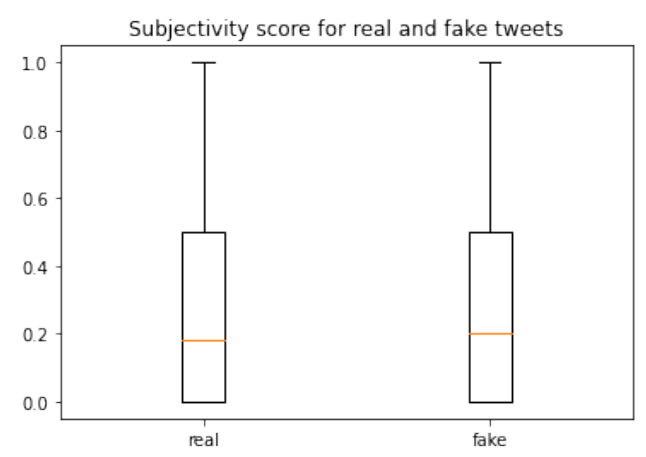

(b) Subjectivity score

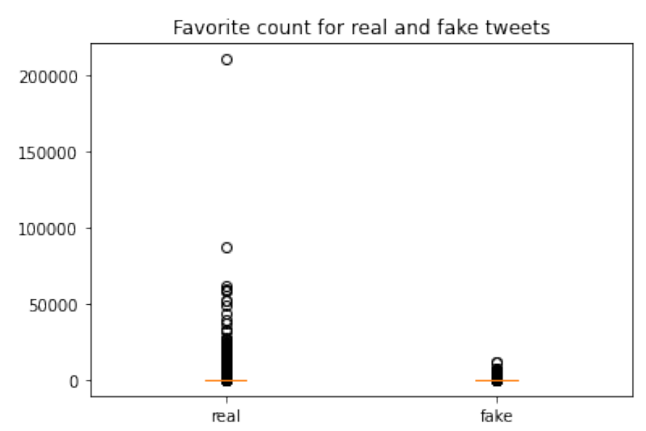

(d) Favorite count

Figure 3. Statistics on sentiment and subjectivity score, as well as on retweet and favorite counts.

Table 1. Actual distribution of tweets in the sample drawn from sources known to contain fake news.

\begin{tabular}{cc}
\hline Category & Amount \\
\hline Fake news & $15.0 \%$ \\
Real news & $40.0 \%$ \\
No news & $26.7 \%$ \\
Unclear & $18.3 \%$ \\
\hline
\end{tabular}

\subsection{Small-Scale Evaluation Dataset}

For creating a hand-labeled gold standard, we used 116 tweets from the politifact web site that were classified as fake news by expert journalists (see above). Those were used as positive examples for fake news tweets. Note that the sources of those tweets are not sources that have been used in the training set. For generating negative examples, and in order to arrive at a non-trivial classification problem, we picked those 116 tweets which were the closest to the fake news tweets in the trustworthy class according to TF-IDF and cosine similarity. By this, we created a balanced gold standard for evaluation of 232 tweets classified as real and fake news.

The rationale for this approach instead of using explicit real news (e.g., from politifact) is not to overly simplify the problem. By selecting a random set of real and fake news each, it is likely to end up with topically unrelated tweets, also since fake news do not spread equally across all news topics. In that case, a classifier could simply learn to distinguish the topics instead of distinguishing fake from real news.

In order to eliminate overfitting effects, we removed the 116 tweets used as negative examples from the training dataset before training our classification models. 


\subsection{Evaluation Scenarios}

We consider two different evaluation scenarios. Scenario 1 only considers the tweet as such. Here, we examine the case where we have a tweet issued from an account for which there is no additional information, e.g., a newly created account.

Scenario 2 also includes information about the user account from which the tweet was sent. Since including as much information as possible will likely improve the results, we expect the mere results of Scenario 2 to be better than those in scenario 1 . However, the Scenario 2 is only applicable for user accounts that have been active for a certain period of time, whereas Scenario 1 has no such constraints.

\section{Approach}

We model the problem as a binary classification problem. Our approach is trained on the large-scale, noisy dataset, using different machine learning algorithms. All of those methods expect the representation of a tweet as a vector of features. Therefore, we use different methods of extracting features from a tweet. We consider five different groups of features: user-level features, tweet-level features, text features, topic features, and sentiment features. For the feature engineering, we draw from previous works that extract features from tweets for various purposes $[7,16,18,19,25-28]$.

The overall approach is depicted in Figure 4. A human expert labels a list of sources, which are then used to crawl tweets for those sources. These crawled tweets then serve as input to a machine learning classifier as examples labeled by weak supervision, and are enriched by different feature generation strategies.

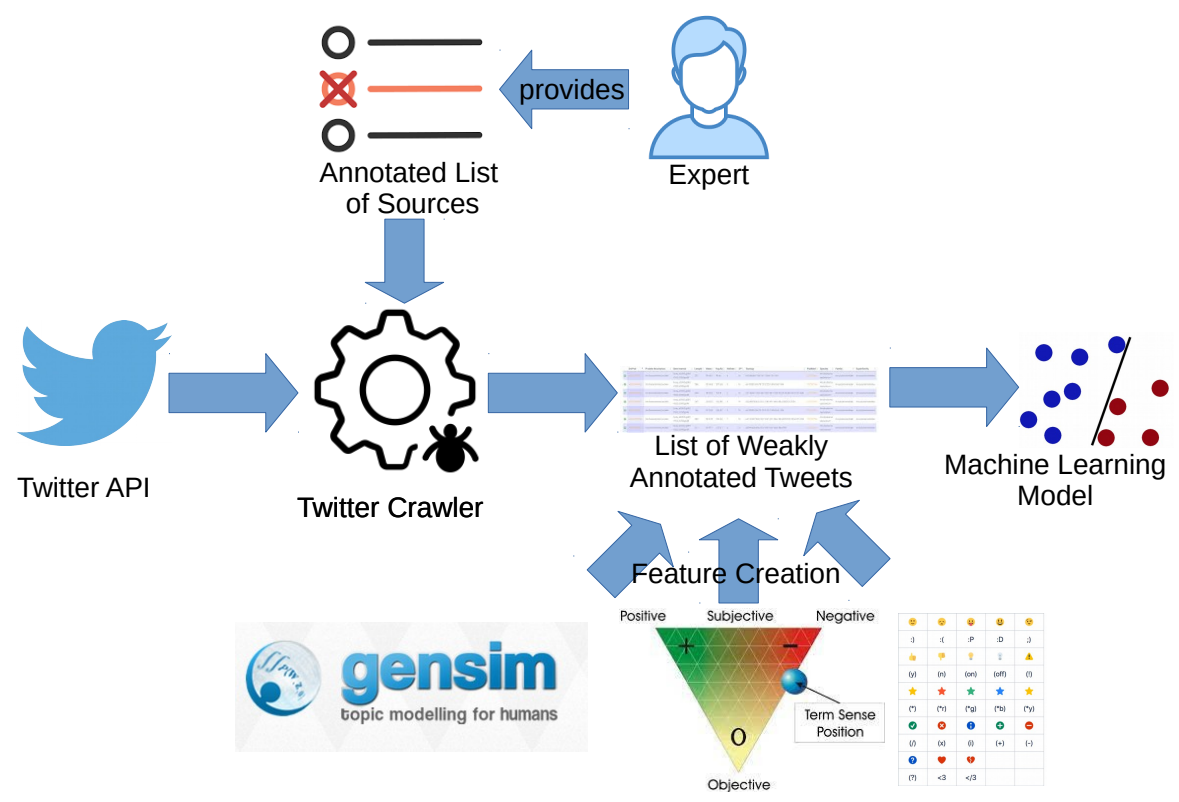

Figure 4. Schematic depiction of the overall approach.

\subsection{User-Level Features}

For the user, we first collect all features that the Twitter API (https: / / developer.twitter. $\mathrm{com} / \mathrm{en} / \mathrm{docs}$ /api-reference-index, accessed on 26 April 2021) directly returns for a user, e.g., the numbers of tweets, followers, and followees, as well as whether the account is a verified account or not (see https://help.twitter.com/en/managing-your-account/abouttwitter-verified-accounts, accessed on 26 April 2021).

In addition to the user-level features that can be directly obtained from the Twitter API, we create a number of additional features, including:

- Features derived from the tweet time (e.g., day, month, weekday, time of day);

- features derived from the user's description, such as its length, usage of URLs, hashtags, etc.; 
- features derived from the user's network, e.g., ratio of friends and followers;

- features describing the tweet activity, such as tweet, retweet, and quote frequency, number of replies, and mentions, etc.;

- features describing the user's typical tweets, such as ratio of tweets containing hashtags, user mentions, or URLs.

In total, we create 53 user-level features. Those are depicted in Tables A1 and A2 in the Appendix A.

\subsection{Tweet-Level Features}

For tweet-level features, we again first collect all information directly available from the API, e.g., number of retweets and retweets, as well as whether the tweet was reported to be sensitive (see https://help.twitter.com/en/safety-and-security/sensitive-media, accessed on 26 April 2021).

In addition, we create a number of additional features characterizing a tweet, e.g.,

- features characterizing the tweet's contents, such as URLs, hashtags, user mentions, and emojis. For emojis, we also distinguish face positive, face negative, and face neutral emojis (http:/ / unicode.org/emoji/charts/full-emoji-list.html, accessed on 20 May 2017, and http:/ / datagenetics.com/blog/october52012/index.html, accessed on 9 May 2017);

- features characterizing the tweet's language, such as punctuation characters, character repetitions, and ratio of uppercase letters;

- linguistic features, such as ratio of nouns, verbs, and adjectives (using WordNet [29] and NLTK [30], number of slang words, determined using Webopedia (http:/ / www. webopedia.com/quick_ref/Twitter_Dictionary_Guide.asp, accessed on 27 April 2021) and noslang.com (http:/ / www.noslang.com/dictionary/, accessed on 27 April 2021), and existence of spelling mistakes (determined using the pyenchant (https:/ / pypi.org/ project/pyenchant/, accessed on 28 April 2021) library).

In total, we create 69 tweet-level features. The full list of features is depicted in Tables A3 and A4. In order to make the approach applicable in real-time scenarios and be able to immediately classify new tweets, we remove time-dependent attributes (i.e., number of retweets and number of favorites).

\subsection{Text Features}

The features above do not consider the actual contents of the tweet. For representing the textual contents of the tweet, we explored two alternatives: a bag of words (BOW) model using TF-IDF vectors, and a neural Doc2vec model [31] trained on the corpus. For the $\mathrm{BOW}$ approach, we explored the use both of word unigrams and word bigrams. The results of an initial experiment using different learners in their default configuration, and running cross validation on the training set, are depicted in Table 2. It can be observed that unigrams work better than bigrams for BOW models, most likely due to the high dimensionality of the latter. 
Table 2. The performances of the learners with a bag-of-words model with 500 terms for unigrams and 250 terms for bigrams. The best performance for unigrams and biagrams is marked in bold.

\begin{tabular}{ccccc}
\hline Learner & Variant & Precision & Recall & F1-Measure \\
\hline Naive Bayes & unigram & 0.3943 & $\mathbf{0 . 6 3 7 6}$ & $\mathbf{0 . 4 8 7 3}$ \\
\hline Decision Tree & unigram & 0.4219 & 0.4168 & 0.4193 \\
\hline SVM & unigram & 0.5878 & 0.1559 & 0.2465 \\
\hline Neural Network & unigram & 0.4471 & 0.3951 & 0.4195 \\
\hline XGBoost & unigram & $\mathbf{0 . 6 6 3 0}$ & 0.0641 & 0.1170 \\
\hline Random Forest & unigram & 0.5226 & 0.4095 & 0.4592 \\
\hline Naive Bayes & bigram & $\mathbf{0 . 4 4 8 6}$ & 0.1320 & 0.2040 \\
\hline Decision Tree & bigram & 0.4139 & $\mathbf{0 . 3 1 7 8}$ & $\mathbf{0 . 3 5 9 5}$ \\
\hline SVM & bigram & 0.5930 & 0.1029 & 0.1754 \\
\hline Neural Network & bigram & 0.3935 & 0.1414 & 0.2081 \\
\hline XGBoost & bigram & 0.4083 & 0.0596 & 0.1040 \\
\hline Random Forest & bigram & 0.4450 & 0.2945 & 0.3544 \\
\hline
\end{tabular}

For doc2vec, two variants exist. On the one hand, the Continuous Bag-of-words model (CBOW) predicting a word at hand based on its context. The context is represented in a bag-of-words fashion, thus the order of the words is not considered. On the other hand, the Continuous Skip-gram model which predicts the surrounding words given a word [32]. Doc2Vec does not only use word vectors, but also paragraph vectors which represent a text of arbitrary length rather than single words. Word vectors are shared across paragraphs whereas paragraph vectors are only shared across the contexts within a paragraph. With Doc2Vec, it is possible to represent texts of variable length with a vector of a fixed size which is usually much smaller than the vocabulary size. This vector can then be used as features for tasks requiring a two dimensional text representation like the one in this paper. Again, two frameworks can be applied to achieve the vectors. The Distributed Memory Model (PV-DM) is similar to CBOW, but also includes a paragraph matrix in addition to the word vectors which then are used to predict a word in the context. Similar to the skip-gram model for Word2Vec, the Distributed Bag-of-words for paragraph vectors (PV-DBOW) predicts words from a small window given the paragraph matrix. The order of the words in this window is not considered [31]. The Python library gensim (https: / / radimrehurek.com/gensim/, accessed on 7 June 2017) was used to create the vectors. As an input for Doc2Vec, preprocessing stage five was considered. This stage also includes stopwords. However, Doc2Vec is able to handle them through the sample parameter, which randomly downsamples higher-frequency words (https: / /radimrehurek. com/gensim/models/doc2vec.html, accessed on 7 June 2017).

While the BOW model yields a fixed number of features (since each possible word in the corpus is regarded as a single feature), we can directly influence the number of features created by Doc2vec. We have experimented with 100, 200, and 300 dimensions, training the model using the gensim Python library (https: / / radimrehurek.com/gensim/, accessed on 7 June 2017), and using both the Distributed Memory (DM) and the Distributed Bag of Words (DBOW) variant. In an initial experiment, running cross validation on the training set, DBOW constantly outperformed DM, but the number of dimensions for which the best results were achieved varied across the learning algorithms, as depicted in Figure 5. Comparing the results to those obtained with BOW above, we can observe that doc2vec yields superior results. Hence, we used doc2vec for text representation in the subsequent experiments, using the best performing configuration for each learning algorithm from the initial experiments (Interaction effects of the parameter settings for doc2vec and the learning algorithm were not analyzed due to the size of the combined parameter search space). 

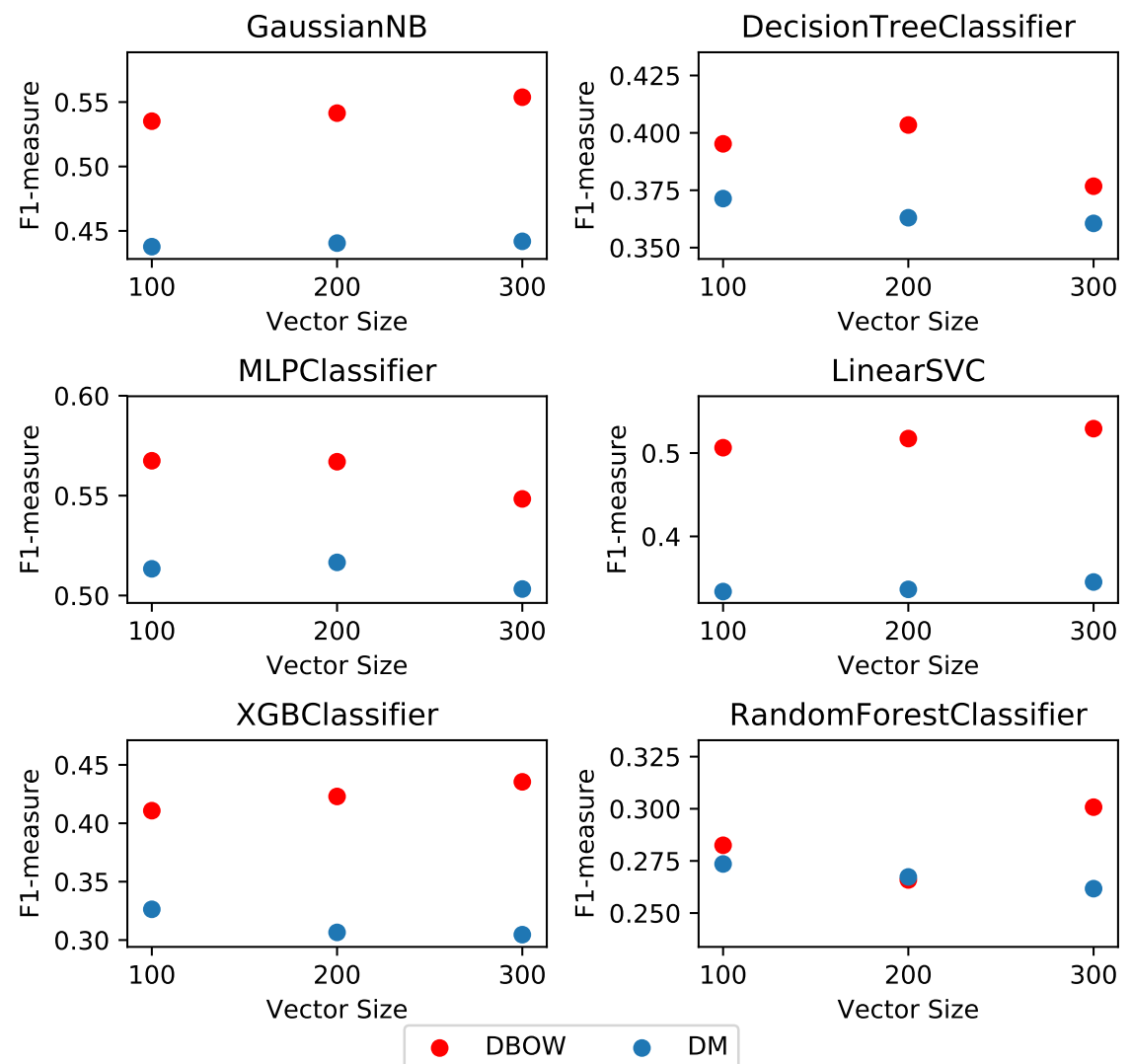

Figure 5. F1 scores using different dimensions for Doc2vec, using DBOW (in blue) and DM (in red).

\subsection{Topic Features}

Since the topic of a tweet may have a direct influence on the fake news classification, as some topics are likely more prone to fake news than others, we also apply topic modeling for creating features from the tweets. Again, we train topic models using the gensim library.

Latent Dirichlet Allocation (LDA) is a three-level hierarchical Bayesian model of a set of documents. Each document is modeled as a mixture of a set of $K$ topics. In turn, each topic is modeled through a distribution over words [33]. The number of topics, i.e., $K$, is a user defined number. In a nutshell, the algorithm first assigns a random topic to each word in the corpus and then iteratively improves these guesses based on the probability for a topic, given a word and a document until a convergence criteria is met. As a simplified example, assume that LDA was configured to discover two topics $t_{0}$ and $t_{1}$ from ten documents, i.e., $K=2$. The output of LDA is then, for instance, that the first document is $80 \%$ about topic $t_{0}$, whereas another document is $40 \%$ about $t_{0}$ and $40 \%$ about topic $t_{1}$. These topics $t_{0}$ and $t_{1}$ are then in turn built from words in the documents. For example, topic $t_{0}$ is built $50 \%$ from a word $w_{0}, 30 \%$ from a word $w_{1}$ and so on. The LDA implementation for online learning was similar to Doc2Vec chosen from the gensim library. The advantage of using an online instead of a batch implementation is that documents can be processed as a stream and discarded after one look. Hence, documents do not need to be stored locally, which in turn is memory efficient [34].

One of the major disadvantages of LDA is that $K$, i.e., the number of topics is a mandatory user specified parameter. Especially when a fixed number of topics is hard to determine as it is for news tweets, setting the best value is not an easy task. The Hierarchical Dirichlet Process (HDP) proposed by [35] solves this problem. It is used to generalize LDA and determines the number of clusters directly from the data. In LDA, a topic is represented by a probability distribution across a set of words and a document by a probability distribution across a finite set of topics. In the nonparametric version, also 
referred to as HDP-LDA, the topics do not come from a finite set but they are linked, so that the same topic can appear in different documents [36]. Again, the online implementation from the gensim library was used. It is inspired by the one for LDA and thus provides the same benefits. In previous research, it has been demonstrated on two text collections that the HDP outperforms the online LDA model according to the per-word likelihood [37].

We trained both a Latent Dirichlet Allocation model (LDA) on the whole dataset, varying the number of topics between 10 and 200 in steps of 10, as well as a Hierarchical Dirichlet Process (HDP) model. As for the text representations with BOW and doc2vec, we conducted a prestudy with different classifiers in their standard configurations, and cross validation on the training set. As shown in Figure 6, LDA constantly outperformed HDP, but the number of topics at which the best results are achieved again varies between learners.
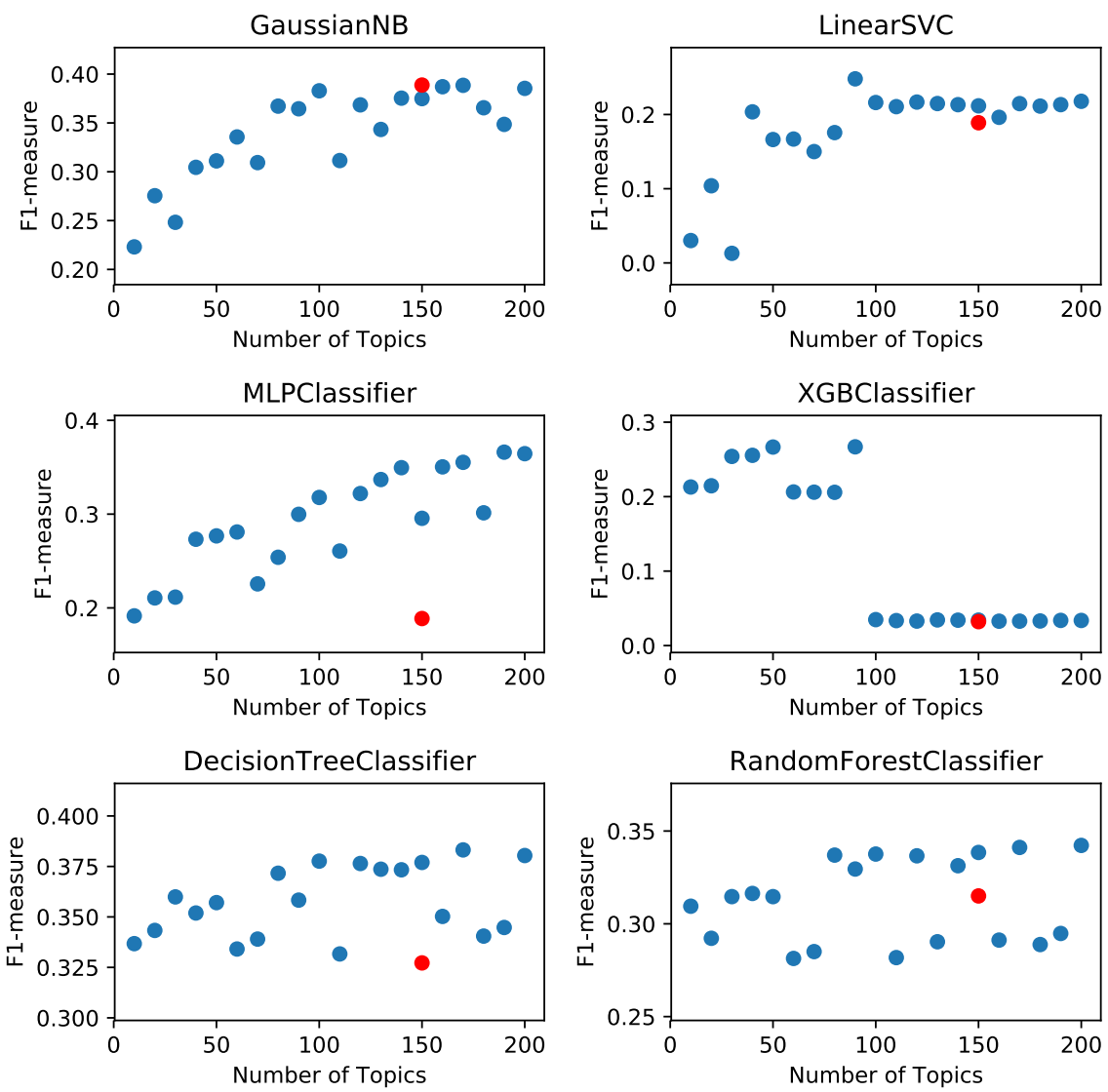

Figure 6. F1 scores using different numbers of topics for LDA with different learning algorithms (in blue), and the results for HDP (in red).

\subsection{Sentiment Features}

The polarity of a tweet can be assessed in terms of positivity, neutrality, or negativity. Additionally, a lemmatization was performed to get a base form of the words. The meaning of words depend on the semantic of the sentence, and thus the context. Hence, the first step was to identify this meaning. In NLP, this task is called Word Sense Disambiguation. One of the most popular algorithms for the disambiguation of word senses is the Lesk algorithm. This algorithm uses word dictionaries with explanations for each word's sense. Words from the sense definition are then crosschecked for overlaps with words in the sense definition of the other words in context. The sense with the highest overlap is then chosen for each word in context [38]. In the NLTK implementation, which was used in this paper, WordNet is used as a dictionary. In that implementation, part-of-speech tags can be included for disambiguation as well. To determine the sentiment of a tweet, SentiWordNet was then 
used to get a positive and negative score for every single word in the context. SentiWordNet is a publicly available lexicon with the annotation of all synsets in WordNet according to their positivity, negativity, and neutrality [39]. All nouns, verbs, adjectives, and adverbs were evaluated, and the difference of positive and negative sentiment was calculated as a sentiment score for each word.

On Twitter, sentiment is not only expressed in words, but also by using emoticons. At this point, emoticons have not yet been considered. Therefore, an emoticon dictionary for Unicode emojis [40], as well as one for ASCII emoticons [41], were used here to look up the sentiment of the emoticons. After all tokens in the sentence have been scored, the tweet's score was calculated by summing up the individual scores. Through summing up the sentiments, the sum's lower and upper bound is limited by the number of words in a tweet, and thus the 140 character limitation. In a tweet with 20 sentiment words of which all are positive, i.e., each word has a score of +1 , the sentiment sum would be 20 . If in another tweet there is only one positive word, the sum would be much lower. It is clear that in this example the tweet with more sentiment words also gets higher scores even if both are completely positive. Therefore the sum was divided by the number of sentiment words in the tweet to get the average sentiment score. Sentiment is usually given in a range of -1 to 1 . However, since many machine learning classifiers operate better on features in a $[0,1]$ interval, the sentiment score was normalized to a range from 0 to 1 , i.e., tweets with a score of 0.5 are completely neutral.

In addition to the previous approach, an implementation from the TextBlob (http: / / textblob.readthedocs.io/en/dev/index.html, accessed on 27 April 2021) library was also considered. This implementation is a lexicon approach as well. It is based on the web mining module Pattern, which was proposed by [42]. Compared to SentiWordNet, Pattern does not only provide scores for polarity, but also a degree of subjectivity. Polarity scores span from -1 , which is very negative, to 1 , which is very positive. Objective words get a score of 0 , whereas highly subjective words like handsome get a score of 1 . In Pattern, words were manually annotated based on the triangle representation, which is shown in Figure 7. The authors claim that "[...] more positive/negative adjectives are also more subjective [but] not all subjective adjectives are necessarily positive or negative [...]" [43]. Although the paper is primarily about Dutch language, the proposed English word annotation showed good results on their test data, too. Unfortunately, the dictionary only scores words, and thus does not include emoticons. Nevertheless, the TextBlob implementation was used as an estimator for subjectivity. URLs, user mentions, hashtags, and punctuation were removed and the remaining text was passed on to the library which then returned a subjectivity score for the text. The tweet feature called subjectivity_score refers to this value.

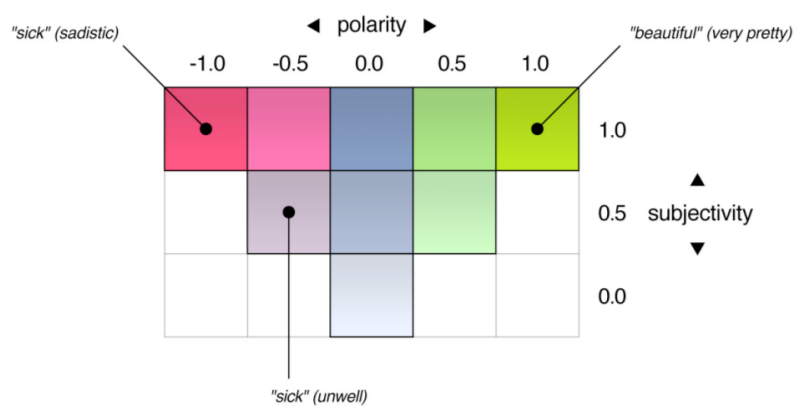

Figure 7. Representation of polarity and subjectivity scores by [43].

Polarity and subjectivity scores account for eight additional features. Table A5 in the Appendix A gives an overview of those features.

\subsection{Feature Scaling and Selection}

The resulting feature set combining all of the above strategies is fairly large, hence, we expect performance gains from dimensionality reduction or feature selection. We explored 
three different options here: setting a minimum variance threshold, recursive elimination using the Gini index as an importance criterion, and recursive elimination using mutual information [44]. The first removes all features which do not have a minimum variance (in our experiments, we used a minimum variance of 0.005), the latter two recursively eliminate the lowest ranked features according to the respective importance measure until the performance drops.

For machine learning approaches that are sensitive to scales, such as Support Vector Machines, we standardize all features first, using a z-transformation in a preprocessing step.

\subsection{Learning Algorithms and Parameter Optimization}

As learning algorithms, we use Naive Bayes, Decision Trees, Support Vector Machines (SVM), and feed-forward Neural Networks as basic classifiers. Moreover, we use two ensemble methods known to usually work well, i.e., Random Forest and XGBoost, as well as voting among all of those methods in two flavors (simple majority voting, and weighted voting based on the classifiers' confidences).

While Naive Bayes and Decision Trees were used in their standard configuration, we performed some parameter optimization for the other classifiers, using a 10\% stratified sample of our training dataset. For SVM, we optimized the parameters $C$ and $\gamma$ as described by [45]. For Neural Networks, we follow the observation by [46] that one hidden layer is sufficient for most classification problems. We optimized the number of neurons in the hidden layer along with many other parameters, such as activation function, learning rate, regularization penalty, and the maximum number of training epochs, using random search as suggested by [47]. Finally, for Random Forests and XGBoost, we pick the number of trees to be learned by simple grid search.

\section{Evaluation}

We evaluate our approach in different settings. First, we perform cross-validation on our noisy training set; second, and more importantly, we train models on the training set and validate them against a manually created gold standard (The code and data are available at https:/ / github.com/s-helm/TwitterFakeNews (accessed on 27 April 2021). Moreover, we evaluate two variants, i.e., including and excluding user features. The rationale of the latter is to simulate two use cases: assessing a tweet from a known user account, and assessing a tweet from a new user account.

In order to further analyze the benefit of our dataset, we also run a cross validation on the evaluation set, and we explore how distributional features generated by utilizing a large-scale dataset (in particular: text and topic features) can be injected into a clean, but smaller training set.

Since the original training set was labeled by source, not by tweet, the first setting evaluates how well the approach performs on the task of identifying fake news sources, whereas the second setting evaluates how well the approach performs on the task of identifying fake news tweets-which was the overall goal of this work. It is important to note that the fake news tweets come from sources that have not been used in the training dataset.

\subsection{Setting 1: Cross-Validation on Training Dataset}

To analyze the capabilities of the predictive models trained, we first perform crossvalidation on the training dataset. Due to the noisy nature of the dataset, the test dataset also carries noisy labels with the same characteristics as the training dataset, and thus, we expect the results to over-estimate the actual performance on a correctly labeled training dataset. Hence, the results depict an upper bound of our proposed weak supervision method.

The results are shown in Tables 3 and 4. Not surprisingly, adding information on the user clearly improves the results. We can observe that the best results are achieved using XGBoost, leading to an F1 score on the fake news class of 0.78 and 0.94 , respectively. 
Although voting using all base classifiers improves over the results of XGBoost alone, the improvement is rather marginal.

Table 3. Cross validation results with tweet features only, and number of features used (\#F). Abbreviations: Learners-Naive Bayes (NB), Decision Tree (DT), Neural Network (NN), Support Vector Machine (SVM), XGBoost (XGB), Random Forest (RF); Feature Selection (FS), Voting (V), Weighted Voting (WV)-Variance Threshold (V), Recursive Elimination with Mutual Information (MI) and with Gini Index (G). The best results are marked in bold.

\begin{tabular}{cccccc}
\hline Learner & \#F & FS & Prec. & Rec. & F1 \\
\hline NB & 409 & V & 0.7024 & 0.6080 & 0.6518 \\
\hline DT & 845 & - & 0.6918 & 0.6401 & 0.6649 \\
\hline NN & 463 & MI & 0.8428 & 0.6949 & 0.7618 \\
\hline SVM & 565 & MI & 0.8492 & 0.6641 & 0.7453 \\
\hline XGB & 106 & G & $\mathbf{0 . 8 6 7 2}$ & $\mathbf{0 . 7 0 1 8}$ & $\mathbf{0 . 7 7 5 8}$ \\
\hline RF & 873 & G & 0.8382 & 0.6494 & 0.7318 \\
\hline V & - & - & 0.8650 & 0.7034 & 0.7759 \\
\hline WV & - & - & 0.8682 & 0.7039 & $\mathbf{0 . 7 7 7 5}$ \\
\hline
\end{tabular}

Table 4. Cross validation results with tweet and user features. The best results are marked in bold.

\begin{tabular}{cccccc}
\hline Learner & $\# \mathbf{F}$ & FS & Prec. & Rec. & F1 \\
\hline NB & 614 & V & 0.8704 & 0.8851 & 0.8777 \\
\hline DT & 34 & G & 0.8814 & 0.9334 & 0.9067 \\
\hline NN & 372 & V & 0.8752 & 0.8861 & 0.8806 \\
\hline SVM & 595 & MI & $\mathbf{0 . 9 3 1 5}$ & 0.7727 & 0.8447 \\
\hline XGB & 8 & G & 0.8946 & $\mathbf{0 . 9 5 8 9}$ & $\mathbf{0 . 9 2 5 6}$ \\
\hline RF & 790 & G & 0.8923 & 0.8291 & 0.8596 \\
\hline V & - & - & 0.9305 & 0.9416 & $\mathbf{0 . 9 3 6 0}$ \\
\hline WV & - & - & 0.9274 & 0.9384 & 0.9329 \\
\hline
\end{tabular}

\subsection{Setting 2: Validation Against Gold Standard}

As discussed above, the more important setting validates the approach using a manually annotated gold standard. Since that gold standard dataset was collected independently from the training dataset, and is never used for training, feature selection, or parameter optimization, we can safely state that our approach is not overfit to that dataset.

For the feature sets, feature selection methods, and parameter settings, we used the setups that worked best in the cross validation settings. More precisely, the feature sets and feature selection methods are those depicted in Tables 3 and 4 .

The results of that evaluation are shown in Tables 5 and 6 . In contrast to the results in cross validation, the neural network learner performs best in that scenario, and voting across all learners does not improve the results. 
Table 5. Results on manually labeled gold standard, using tweet features only. The best results are marked in bold.

\begin{tabular}{cccc}
\hline Learner & Precision & Recall & F1-Measure \\
\hline NB & 0.6835 & 0.4655 & 0.5538 \\
\hline DT & 0.7895 & 0.6466 & 0.7109 \\
\hline NN & 0.7909 & $\mathbf{0 . 7 5 0 0}$ & $\mathbf{0 . 7 6 9 9}$ \\
\hline SVM & 0.7961 & 0.7069 & 0.7489 \\
\hline XGB & 0.8333 & 0.6897 & 0.7547 \\
\hline RF & $\mathbf{0 . 8 7 2 1}$ & 0.6466 & 0.7426 \\
\hline V & 0.8200 & 0.7069 & 0.7593 \\
\hline WV & 0.8478 & 0.6724 & 0.7500 \\
\hline
\end{tabular}

Table 6. Results on manually labeled gold standard, using both tweet and user features. The best results are marked in bold.

\begin{tabular}{cccc}
\hline Learner & Precision & Recall & F1-Measure \\
\hline NB & 0.8333 & $\mathbf{0 . 9 0 5 2}$ & 0.8678 \\
\hline DT & 0.8721 & 0.6466 & 0.7426 \\
\hline NN & $\mathbf{0 . 9 1 1 5}$ & 0.8879 & $\mathbf{0 . 8 9 9 6}$ \\
\hline SVM & 0.8942 & 0.8017 & 0.8455 \\
\hline XGB & 0.8679 & 0.7931 & 0.8288 \\
\hline RF & 0.8713 & 0.7586 & 0.8111 \\
\hline V & 0.8870 & 0.8793 & 0.8831 \\
\hline WV & 0.8761 & 0.8534 & 0.8646 \\
\hline
\end{tabular}

Table 7 summarizes the best achieved results for each of the four settings. It is remarkable that the results are not much worse than those achieved in cross validation: with tweet features only, the best F1 score achieved is 0.77 (compared to 0.78), with both tweet and user features, the best F1 score is 0.90 (compared to 0.94). This proves that our approach of using a large-scale, noisy training dataset is actually valid and yields results of remarkable quality, even though the classifier employed was originally trained on a different task.

Table 7. Results summary, depicting the best F1 score achieved for each task (fake news source and fake news tweet detection), as well as for each feature group (tweet level features only and tweet and source features).

\begin{tabular}{ccc}
\hline & \multicolumn{2}{c}{ Identifying Fake News... } \\
\hline Tweet features only & Sources & Tweets \\
\hline Tweet and user features & 0.7775 (Table 3) & 0.7699 (Table 5) \\
\hline
\end{tabular}

\subsection{Comparison to Training on Manually Labeled Data}

In order to be able to better judge the results above, we also ran a set of experiment where we use cross validation on the evaluation set, i.e., we use the manually labeled data as training signal. The goal of this experiment is to analyze how close the quality using weak supervision would get to results achieved through human expert labeling, which is expected to yield superior results. 
Here, we distinguish two cases:

1. In the first setup, we have re-created all content features for the manually labeled dataset (On the manually labeled dataset, LDA was not able to generate meaningful results due to the very small dataset size. Hence, we have left out the LDA based features in the first setup).

2. In the second setup, we used the distributional text and topic features (doc2vec, LDA) created from the weakly supervised set.

The latter case can be considered a combined setup, where information from the large dataset obtained through weak supervision was used to improve the results on the manually labeled dataset.

The parameter settings were the same as in the experiments on the large-scale training data. The results are depicted in Tables 8 and 9. They show that the F1 scores are in the same range when using the manually labeled tweets as when using the ones labeled through weak supervision - when using tweet features only, training on the manually labeled data works slightly better, when using all features, training on the weak supervision set works slightly better. This supports our claim that our methodology for generating training data is valid in the sense that it yields datasets of comparable quality in downstream classification tasks.

Table 8. Cross validation results on manually labeled gold standard, using tweet features only. We distinguish a setup with re-created features for the manually labeled dataset, and re-using features from the large dataset. The best results are marked in bold.

\begin{tabular}{ccccccc}
\hline & \multicolumn{3}{c}{ Re-Created Features } & \multicolumn{3}{c}{ Features from Large Set } \\
\hline Learner & Precision & Recall & F1-Measure & Precision & Recall & F1-Measure \\
\hline NB & 0.5912 & 0.6983 & 0.6403 & 0.4425 & 0.4310 & 0.4367 \\
\hline DT & 0.6944 & 0.6466 & 0.6696 & 0.7156 & 0.6724 & 0.6933 \\
\hline NN & 0.7500 & $\mathbf{0 . 8 0 1 7}$ & 0.7750 & 0.6640 & 0.7155 & 0.6888 \\
\hline SVM & 0.7311 & 0.7500 & 0.7404 & $\mathbf{0 . 8 5 8 5}$ & 0.7845 & $\mathbf{0 . 8 1 9 8}$ \\
\hline XGB & $\mathbf{0 . 8 0 0 0}$ & 0.7586 & $\mathbf{0 . 7 7 8 8}$ & 0.7797 & $\mathbf{0 . 7 9 3 1}$ & 0.7863 \\
\hline RF & 0.7524 & 0.6810 & 0.7149 & 0.7938 & 0.6638 & 0.7230 \\
\hline
\end{tabular}

Table 9. Cross validation results on manually labeled gold standard, using user and tweet features. We distinguish a setup with re-created features for the manually labeled dataset, and re-using features from the large dataset. The best results are marked in bold.

\begin{tabular}{ccccccc}
\hline & \multicolumn{3}{c}{ Re-Created Features } & \multicolumn{2}{c}{ Features from Large Set } \\
\hline Learner & Precision & Recall & F1-Measure & Precision & Recall & F1-Measure \\
\hline NB & 0.8235 & 0.7241 & 0.7706 & 0.5619 & 0.6983 & 0.6255 \\
\hline DT & 0.8750 & 0.9052 & $\mathbf{0 . 8 8 9 8}$ & 0.8571 & 0.8793 & 0.8681 \\
\hline NN & 0.8547 & 0.8621 & 0.8584 & 0.8110 & 0.8879 & 0.8477 \\
\hline SVM & 0.8584 & 0.8362 & 0.8472 & $\mathbf{0 . 9 2 9 9}$ & $\mathbf{0 . 9 1 3 8}$ & $\mathbf{0 . 9 2 1 7}$ \\
\hline XGB & $\mathbf{0 . 8 9 4 7}$ & 0.8793 & 0.8870 & 0.8983 & $\mathbf{0 . 9 1 3 8}$ & 0.9059 \\
\hline RF & 0.8618 & $\mathbf{0 . 9 1 3 8}$ & 0.8870 & 0.8522 & 0.8448 & 0.8485 \\
\hline
\end{tabular}

In our second setup, we use the distributional features created on the large-scale dataset. Here, our intuition is that approaches such as LDA and doc2vec yield good results only when trained on larger corpora. When using those features for classification with the manually labeled data, we obtain the best results overall, surpassing both the setup with manually labeled data only and the setup with labeling through weak supervision only by 3-4 percentage points in F1 score. 


\subsection{Feature Importance}

Another question we look into is the relative importance of each feature for the task of fake news detection. Since XGBoost can also compute an importance score for each feature, we could directly plot the most relevant features for the two evaluation scenarios, as depicted in Figures 8 and 9.

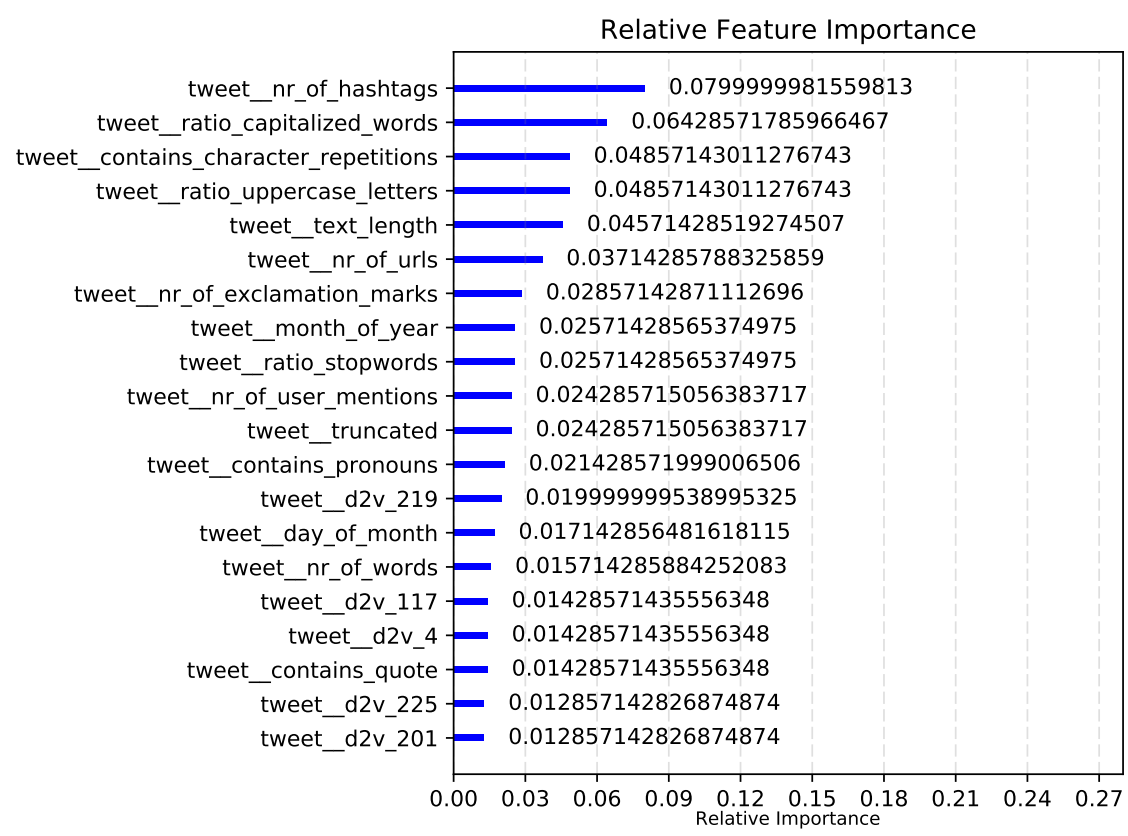

Figure 8. Feature importance for Scenario 1 (tweet level features only), as computed by XGBoost.

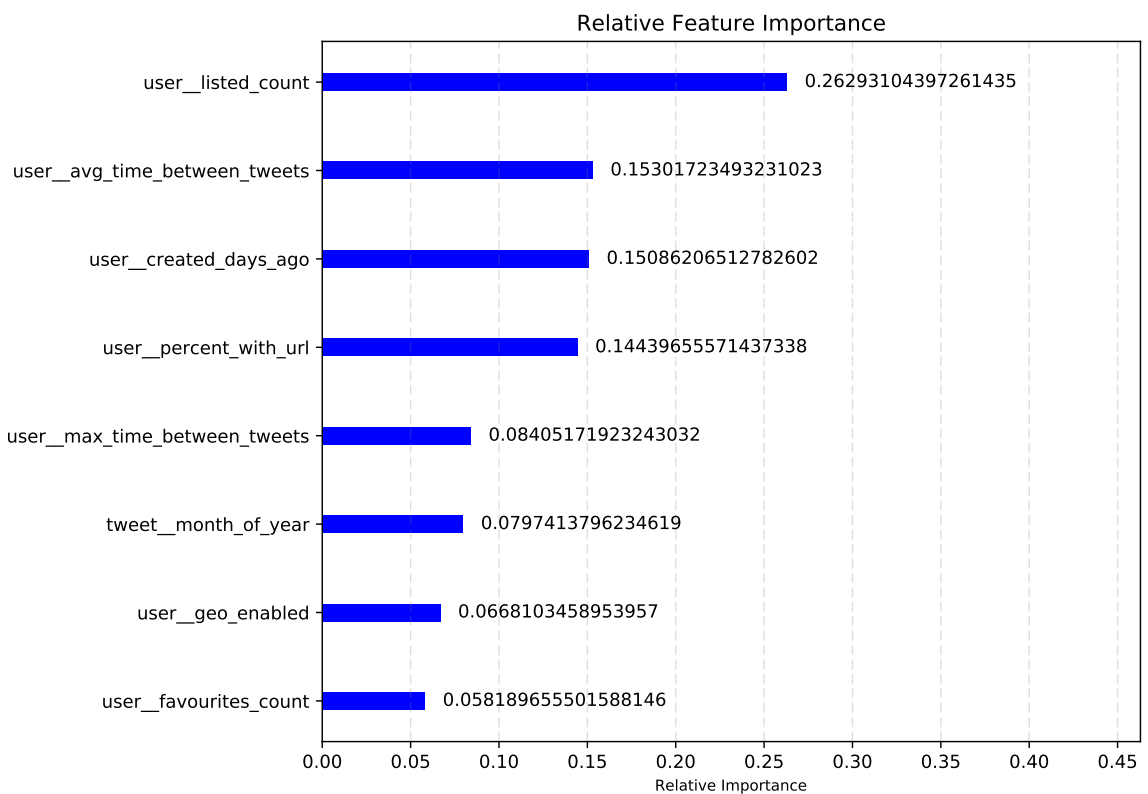

Figure 9. Feature importance for Scenario 2 (tweet and user level features), as computed by XGBoost.

The first observation to be made is that user features, if made available as in Scenario 2, receive a much higher weight. The explanation for this is two fold: first, it is an effect of the specific type of weak supervision used, since the original labels are labels for tweet sources, and each tweet source corresponds to a Twitter user. Second, there is obviously a strong correlation between user behavior (e.g., tweet frequency, usage of URLs) and the likelihood 
of a tweet being fake news. The network of a user also matters: the strongest indicator is the containment of a user in lists created by other users.

When looking at tweet level features only, as in Scenario 1, more features with lower weights are selected by XGBoost, indicating that this classification problem is inherently harder. We can see that text surface features, such as capitalization, character repetitions, and exclamation marks play a more crucial role in this respect. Moreover, quite a few doc2vec features for the contents are selected, while topics and sentiment features are not considered helpful by XGBoost.

The fact that the month of the year is selected as a relevant feature is probably an artifact of the dataset distribution, as shown in Figure 1. From this distribution, we can see that the months February-June 2017 have a higher ratio of real to fake news tweets than the other months, which is an effect of the data collection and the rate limitations of the Twitter API, as discussed above.

\section{Conclusions and Outlook}

In this work, we have shown a practical approach for treating the identification of fake news on Twitter as a binary machine learning problem. While that translation to a machine learning problem is rather straight forward, the main challenge is to gather a training dataset of suitable size. Here, we have shown that, instead of creating a small, but accurate hand-labeled dataset, using a large-scale dataset with inaccurate labels yields competitive results.

The advantages of using our approach are two-fold. First, the efforts for creating the dataset are rather minimal, requiring only a few seed lists of trustworthy and nontrustworthy Twitter accounts, which exist on the Web. Second, since the dataset is can be created automatically to a large extent, it can updated at any point of time, thus accounting for recent topics and trends, as well as changes in the social media service (e.g., the change from 140 to 280 characters in Twitter, or the availability of different content via changes in the API (https://developer.twitter.com/en/docs/twitter-api/early-access, accessed on 27 April 2021). In contrast, a hand-labeled dataset might lose its value over time for those reasons. In a practical setup, a fake news detection system based on our approach could continuously collect training data and periodically update its classification models. Moreover, it would allow broadening the scope of the work-while in this work, we have mainly considered political fake news, we could take the same approach to also detect fake news in other domains. Topic classification of tweets would be possible with a similar approach as well-since there are quite a few Twitter accounts which are mainly focused on a particular topic, a training dataset for that task could be sourced with the same approach.

We have shown that our approach yields very good results, achieving an F1 score of 0.77 when only taking into account a tweet as such, and up to 0.9 when also including information about the user account. It is particularly remarkable that the results are not much worse than those achieved for classifying trustworthy and untrustworthy sources (which is actually reflected in the labels for the tweets): with tweet features only, the best F1 score achieved is 0.78 , with both tweet and user features, the best F1 score 0.94 .

To date, we have used features based on the tweets and user accounts, but there are other alternatives as well. For example, for tweets containing a URL, we are currently not collecting any information from and about that URL. The same holds for images and other media in tweets. Second, we only record the number of retweets and likes of a tweet, but do not analyze comments and answers to a tweet, nor any information derived from the user accounts that retweeted a tweet. However, our feature analysis has revealed that the network of users (as manifested, e.g., in the number of user created lists an account is contained in) plays an important role for the classification task at hand. Here, stepping from the level of tweets and users as single entities to considering the entire network of users and tweets would yield new opportunities.

From a machine learning perspective, we have so far taken well-known classification approaches, and shown that they can be applied even in our setting, where the label 
information is noisy (more precisely: where one class is particularly prone to noise). There are quite a few works which theoretically examine the effect of label noise on the learning algorithms and propose specific methods for tailoring learning algorithms for those cases, as well as for cleansing label noise as a preprocessing step [48-50]. Further exploring the application of such techniques will be an interesting aspect of future work in this direction.

In summary, we have shown that the problem of acquiring large-scale training datasets for fake news classification can be circumvented when accepting a certain amount of label noise, which can be used to learn classification models of competitive performance.

Author Contributions: Conceptualization, H.P. and S.H.; methodology, H.P. and S.H.; software, S.H.; validation, H.P. and S.H.; formal analysis, H.P.; investigation, S.H.; resources, S.H.; data curation, S.H.; writing—original draft preparation, S.H.; writing—review and editing, H.P.; visualization, H.P.; supervision, H.P.; project administration, H.P. All authors have read and agreed to the published version of the manuscript.

Funding: This research received no external funding.

Institutional Review Board Statement: Not applicable.

Informed Consent Statement: Not applicable.

Data Availability Statement: Data is available at https://github.com/s-helm/TwitterFakeNews (accessed on 26 April 2021).

Conflicts of Interest: The authors declare no conflict of interest.

\section{Appendix A. Features}

Appendix A.1. User-Level Features

Table A1. Overview of the attributes of a user returned by the Twitter API.

\begin{tabular}{ll}
\hline Feature Name & Description \\
\hline default_profile & Indicates that a user has not changed the theme or background \\
\hline favourites_count & The number of tweets the user liked at the point of gathering \\
\hline followers_count & The number of followers of an account at the point of gathering \\
\hline friends_count & The number of users following the account at the point of gathering \\
\hline geo_enabled & If true, the user has enabled the possibility of geotagging its tweets \\
\hline listed_count & The number of public lists that a user is a member of \\
\hline profile_background_tile & $\begin{array}{l}\text { Indicates whether the URL of the background image should be tiled } \\
\text { when displayed }\end{array}$ \\
\hline profile_use_background_image & Indicates whether the user uses a background image \\
\hline statuses_count & The number of tweets the user published at the point of gathering \\
\hline verified & Indicates whether the account is verified \\
\hline
\end{tabular}


Table A2. Overview of the derived user level features.

\begin{tabular}{|c|c|}
\hline Feature Name & Description \\
\hline has_desc & True if the user uses a description \\
\hline has_location & Flag to indicate whether there is a user-defined location or not \\
\hline desc_contains_hashtags & Specifies if there are hashtags within the description \\
\hline desc_contains_user_mention & Specifies if there are user mentions in the description \\
\hline desc_contains_url & Specifies if there is an URL in the description \\
\hline desc_length & Counts the characters in the description \\
\hline url_length & Specifies the length of the user URL \\
\hline has_url & If true, the user stated a profile URL. \\
\hline has_list & If true, the user was included in at least one list \\
\hline has_favourites & If true, the account has liked tweets during its life time \\
\hline has_friends & If true, the user follows other accounts \\
\hline favourites_per_follower & Ratio between liked tweets and follower count \\
\hline friends_per_follower & Ratio between the number of accounts the account follows and its follower count \\
\hline is_following_more_than_100 & Indicates that the account follows at least 100 Twitter users \\
\hline avg_user_mention_per_tweet & States the average number of user mentions per tweet in the collected data \\
\hline avg_hashtags_per_tweet & States the average number of hashtags per tweet in the collected data \\
\hline avg_urls_per_tweet & States the average number of URLs an account uses in the collected tweets \\
\hline percent_with_url & States the ratio of the collected tweets that contains an URL \\
\hline percent_with_hashtag & States the ratio of the collected tweets that contain a hashtag \\
\hline percent_with_user_mention & States the ratio of the collected tweets that contains a user mention \\
\hline created_days_ago & $\begin{array}{l}\text { The number of days since the creation of the account. Depends on the time this } \\
\text { feature was created. }\end{array}$ \\
\hline created_hour_of_day & The hour the account was created in UTC time \\
\hline avg_post_time & $\begin{array}{l}\text { States the average post time of the user. If present, the UTC offset was taken into } \\
\text { account otherwise the most frequent UTC offset across all collected accounts was } \\
\text { used. }\end{array}$ \\
\hline tweets_per_day & $\begin{array}{l}\text { States the average number of status updates per day. The first and last day of the } \\
\text { user's collected tweets were omitted since they might not be complete. }\end{array}$ \\
\hline tweets_per_week & $\begin{array}{l}\text { States the average number of status updates per week. The first and last week of } \\
\text { the user's collected tweets were omitted since they might not be complete. }\end{array}$ \\
\hline tweets_per_month & $\begin{array}{l}\text { States the average number of status updates per month. The first and last month } \\
\text { of the user's collected tweets were omitted since they might not be complete. }\end{array}$ \\
\hline min_time_between_tweets & States the minimum time between two of the user's tweets in minutes \\
\hline max_time_between_tweets & States the maximum time between two of the user's tweets in minutes \\
\hline median_time_between_tweets & States the median time between two of the user's tweets in minutes \\
\hline avg_time_between_tweets & States the average time between two of the user's tweets in minutes \\
\hline nr_of_retweets & States the number of retweets that were collected from the account \\
\hline nr_of_retweets_per_tweet & $\begin{array}{l}\text { The ratio between the frequency of retweets and the number of tweets in the } \\
\text { collected data }\end{array}$ \\
\hline nr_of_quotes & States the number of quotes that were collected from the account \\
\hline nr_of_quotes_per_tweet & $\begin{array}{l}\text { The ratio between the frequency of quotes and the number of tweets in the } \\
\text { collected data }\end{array}$ \\
\hline nr_of_replies & States the number of replies that were collected from the account \\
\hline nr_of_replies_per_tweet & $\begin{array}{l}\text { The ratio between the frequency of replies and the number of tweets in the } \\
\text { collected data }\end{array}$ \\
\hline has_profile_background_image & States whether a background image is used \\
\hline is_translator_type_regular & If true, the user has type regular in the Twitter translator community \\
\hline tweets_in_different_lang & $\begin{array}{l}\text { If true, the account uses different languages in its tweets. A tweet's languages is } \\
\text { automatically specified by Twitter. }\end{array}$ \\
\hline uses_retweets & If true, the collected data contains retweets by this account \\
\hline uses_quotes & If true, the collected data contains quotes by this account \\
\hline uses_replies & If true, the collected data contains replies by this account \\
\hline url_tld_type & $\begin{array}{l}\text { If false, the user's URL has a generic top level domain otherwise its type is } \\
\text { country-code }\end{array}$ \\
\hline
\end{tabular}




\section{Appendix A.2. Tweet-Level Features}

Table A3. Overview of the status attributes returned by the Twitter API directly adopted as tweet features.

\begin{tabular}{ll}
\hline Feature Name & Description \\
\hline favorite_count & The number of times a tweet has been liked by Twitter users \\
\hline possibly_sensitive & $\begin{array}{l}\text { Indicates that the URL in a tweet may contain sensitive content or media. This is } \\
\text { only present if the tweet contains an URL. }\end{array}$ \\
\hline retweeted_count & The number of times a tweet has been retweeted \\
\hline truncated & Indicates that the tweet's text was truncated because it exceeds the character limit \\
\hline
\end{tabular}

Table A4. Overview of the derived tweet level features.

\begin{tabular}{|c|c|}
\hline Feature Name & Description \\
\hline has_place & $\begin{array}{l}\text { If true, the API returned a place. Places are only associated to a tweet and are not } \\
\text { necessarily the place where a tweet actually was created. }\end{array}$ \\
\hline has_location & If true, the coordinates of the creation location were returned \\
\hline nr_of_urls & $\begin{array}{l}\text { Counts the number of URLs in a tweet. URLs not yet parsed out of the text by Twitter are } \\
\text { included as well. }\end{array}$ \\
\hline contains_urls & States whether a tweet contains URLs or not \\
\hline avg_url_length & States the length of the URL or the average length in case there is more than one URL. \\
\hline url_only & If true, there are no other characters but an URL \\
\hline nr_of_hashtags & Counts the number of hashtags in a tweet \\
\hline contains_hashtags & If true, the tweet contains at least one hashtag \\
\hline nr_of_popular_hashtags & $\begin{array}{l}\text { Counts the number of hashtags in a tweet that are in the top } 100 \text { most frequently used } \\
\text { hashtags across all users. This avoids to include hashtags which are frequent but only } \\
\text { used by a single user. }\end{array}$ \\
\hline contains_popular_hashtag & If true, the tweet contains one of the 100 most frequently used hashtags across all users \\
\hline nr_of_medias & The number of medias the tweet contains which, for example, includes a photo \\
\hline contains_media & If true, the tweet contains media \\
\hline nr_of_user_mentions & States the number of users mentioned in the tweet \\
\hline contains_user_mention & If true, the tweet contains at least one user mention \\
\hline nr_of_unicode_emojis & States the number of unicode emoticons that are in the tweet's text \\
\hline contains_unicode_emojis & If true, the tweet contains at least one unicode emoticon \\
\hline $\begin{array}{l}\text { contains_face_positive } \\
\text { _emojis }\end{array}$ & If true, the tweet contains unicode emoticons from category face positive \\
\hline $\begin{array}{l}\text { contains_face_negative } \\
\text { _emojis }\end{array}$ & If true, the tweet contains unicode emoticons from category face negative \\
\hline $\begin{array}{l}\text { contains_face_neutral } \\
\text { _emojis }\end{array}$ & If true, the tweet contains unicode emoticons from category face neutral \\
\hline nr_of_ascii_emojis & Counts the number of ASCII emoticons in the text \\
\hline contains_ascii_emojis & If true, there are ASCII emoticons in the text \\
\hline contains_stock_symbol & If true, there is at least one stock mention ( $\$$ followed by alphabet characters) in the text \\
\hline nr_of_punctuation & Counts the number of punctuations in a tweet \\
\hline contains_punctuation & If true, the tweet contains punctuation \\
\hline ratio_punctuation_tokens & The ratio of tokens that are actually punctuation \\
\hline nr_of_exclamation_marks & The number of exclamation marks in the tweet \\
\hline contains_exclamation_mark & If true, the tweet contains at least one exclamation mark \\
\hline $\begin{array}{l}\text { multiple_exclamation } \\
\text { marks }\end{array}$ & If true, there are at least two exclamation marks \\
\hline nr_of_question_marks & The number of question marks in the tweet \\
\hline contains_question_mark & If true, the tweet contains at least one question mark \\
\hline multiple_question_marks & If true, there are at least two question marks \\
\hline $\begin{array}{l}\text { contains_character } \\
\text { repetitions }\end{array}$ & If true, a character is followed by at least two repetitions of itself \\
\hline
\end{tabular}


Table A4. Cont.

\begin{tabular}{|c|c|}
\hline Feature Name & Description \\
\hline contains_number & If true, the tweet contains a token that is a numerical \\
\hline contains_quote & If true, there is a quoted text in the tweet \\
\hline day_of_week & The day of the week the tweet was created \\
\hline day_of_month & The day of the month the tweet was created \\
\hline month_of_year & The month the tweet was created \\
\hline am_pm & $\begin{array}{l}\text { If true, the tweet was published after noon. If present, the users UTC offset was } \\
\text { considered otherwise the most frequent offset was used. }\end{array}$ \\
\hline hour_of_day & $\begin{array}{l}\text { The hour the tweet was published in the } 24 \mathrm{~h} \text { format. If present, the users UTC offset was } \\
\text { considered otherwise the most frequent offset was used. }\end{array}$ \\
\hline quarter_of_year & The quarter of the year the tweet was published \\
\hline no_text & $\begin{array}{l}\text { If true, a tweet does not contain text. This is possible when the status update is an URL } \\
\text { only. }\end{array}$ \\
\hline $\begin{array}{l}\text { avg_word_length } \\
\text { text_length }\end{array}$ & $\begin{array}{l}\text { The average word length in the tweet. URLs are not counted. } \\
\text { The length of the text according to the Twitter character counting. User mentions are not } \\
\text { counted. The feature is based on the raw text. }\end{array}$ \\
\hline percent_of_text_used & The ratio of used characters to the 140 possible characters. \\
\hline nr_of_words & $\begin{array}{l}\text { Counts the words a in tweet. This feature is based on preprocessing stage two. } \\
\text { Punctuation is not included into the count. }\end{array}$ \\
\hline nr_of_tokens & $\begin{array}{l}\text { Counts the number of tokens that were generated by the tokenizer. It considers } \\
\text { preprocessing stage three. }\end{array}$ \\
\hline ratio_words_tokens & States the ratio between words and tokens. \\
\hline ratio_uppercase_letters & The ratio of uppercase letters to all cased characters in the tweet's text \\
\hline $\begin{array}{l}\text { is_all_uppercase } \\
\text { contains_uppercase_text }\end{array}$ & $\begin{array}{l}\text { If true, all characters in the raw text are uppercase } \\
\text { If true, the tweet contains a sequence of characters of length five or larger that contain } \\
\text { only uppercase letters }\end{array}$ \\
\hline ratio_capitalized_words & $\begin{array}{l}\text { Counts the number of capitalized words and relates them to the word count. Words with } \\
\text { only capitalized characters are not included in the count for capitalized words. }\end{array}$ \\
\hline ratio_all_capitalized_words & $\begin{array}{l}\text { Ratio of words that contain only capitalized letters to the total number of words. It is } \\
\text { based on preprocessing stage two. }\end{array}$ \\
\hline nr_of_sentences & $\begin{array}{l}\text { Counts the number of sentences. If there is only one sentence, this does not necessarily } \\
\text { mean that this is a complete sentence. Preprocessing stage seven refers to this feature. }\end{array}$ \\
\hline $\begin{array}{l}\text { contains_character } \\
\text { repetitions }\end{array}$ & $\begin{array}{l}\text { If true, a character is followed by at least two repetitions of itself in the raw text from stage } \\
\text { one. }\end{array}$ \\
\hline nr_of_slang_words & $\begin{array}{l}\text { The number of slang words in the tweet from the slang word dictionary. Preprocessing } \\
\text { stage two was used here. }\end{array}$ \\
\hline contains_slang & If true, the tweet contains at least one slang word \\
\hline ratio_adjectives & The ratio of adjectives to the total number of words after POS tagging in stage four \\
\hline ratio_nouns & The ratio of nouns to the total number of words after POS tagging in stage four \\
\hline ratio_verbs & Ratio of verbs to the total number of words after POS tagging in stage four \\
\hline contains_named_entity & If true, a token of the tweet was tagged as a named entity after POS tagging in stage four \\
\hline contains_pronouns & If true, a token of the tweet was tagged as a pronoun after POS tagging in stage four \\
\hline $\begin{array}{l}\text { additional_preprocessed } \\
\text { _is_empty }\end{array}$ & If true, after all text preprocessing steps in stage six no tokens are left \\
\hline $\begin{array}{l}\text { ratio_tokens_before_after } \\
\text { _prepro }\end{array}$ & $\begin{array}{l}\text { The ratio of the number of tokens before preprocessing (stage three) to the number of } \\
\text { tokens afterwards (stage six). Can be greater than one since abbreviations are resolved } \\
\text { after tokenization. }\end{array}$ \\
\hline ratio_stopwords & $\begin{array}{l}\text { The ratio of the tokens from preprocessing stage six where stopwords were removed to } \\
\text { the number of tokens in stage five which includes stopwords }\end{array}$ \\
\hline contains_spelling_mistake & If true, a spelling mistake was found through spell checking \\
\hline
\end{tabular}




\section{Appendix A.3. Sentiment Features}

Table A5. Overview of the features that were created according to the polarity of a tweet.

\begin{tabular}{ll}
\hline Feature Name & Description \\
\hline sentiment_score & The average sentiment of the words in a tweet \\
\hline contains_sentiment & If true, the sentiment score is different from 0.5 \\
\hline nr_pos_sentiment_words & The number of positive words in a tweet \\
\hline nr_neg_sentiment_words & The number of negative words in a tweet \\
\hline nr_of_sentiment_words & The total number of words that got a sentiment score in a tweet \\
\hline ratio_pos_sentiment_words & The ratio of the number of positive words to the number of all sentiment words \\
\hline ratio_neg_sentiment_words & The ratio of the number of negative words to the number of all sentiment words \\
\hline
\end{tabular}

\section{References}

1. Ratkiewicz, J.; Conover, M.; Meiss, M.R.; Gonçalves, B.; Flammini, A.; Menczer, F. Detecting and Tracking Political Abuse in Social Media. ICWSM 2011, 11, 297-304.

2. Ferrara, E.; Varol, O.; Davis, C.; Menczer, F.; Flammini, A. The rise of social bots. Commun. ACM 2016, 59, 96-104. [CrossRef]

3. Shu, K.; Sliva, A.; Wang, S.; Tang, J.; Liu, H. Fake news detection on social media: A data mining perspective. ACM SIGKDD Explor. Newsl. 2017, 19, 22-36. [CrossRef]

4. Allcott, H.; Gentzkow, M. Social Media and Fake News in the 2016 Election; Working Paper 23089; National Bureau of Economic Research: Cambridge, MA, USA, 2017. [CrossRef]

5. Chen, E.; Chang, H.; Rao, A.; Lerman, K.; Cowan, G.; Ferrara, E. COVID-19 misinformation and the 2020 US presidential election. Harv. Kennedy Sch. Misinf. Rev. 2021. [CrossRef]

6. Pennycook, G.; Rand, D.G. Research note: Examining false beliefs about voter fraud in the wake of the 2020 Presidential Election. Harv. Kennedy Sch. Misinf. Rev. 2021. [CrossRef]

7. Deshwal, A.; Sharma, S.K. Twitter sentiment analysis using various classification algorithms. In Proceedings of the 20165 th International Conference on Reliability, Infocom Technologies and Optimization (Trends and Future Directions) (ICRITO), Noida, India, 7-9 September 2016; pp. 251-257. [CrossRef]

8. Selvaperumal, P.; Suruliandi, A. A short message classification algorithm for tweet classification. In Proceedings of the 2014 International Conference on Recent Trends in Information Technology, Chennai, India, 10-12 April 2014; pp. 1-3. [CrossRef]

9. Rosenthal, S.; Nakov, P.; Kiritchenko, S.; Mohammad, S.; Ritter, A.; Stoyanov, V. SemEval-2015 Task 10: Sentiment Analysis in Twitter. In SemEval at NAACL-HLT; Association for Computational Linguistics: Stroudsburg, PA, USA, 2015; pp. 451-463.

10. Zubiaga, A.; Spina, D.; Martinez, R.; Fresno, V. Real-time classification of twitter trends. J. Assoc. Inf. Sci. Technol. 2015, 66, 462-473. [CrossRef]

11. Cole-Lewis, H.; Varghese, A.; Sanders, A.; Schwarz, M.; Pugatch, J.; Augustson, E. Assessing electronic cigarette-related tweets for sentiment and content using supervised machine learning. J. Med Internet Res. 2015, 17, e208. [CrossRef] [PubMed]

12. Yang, S.H.; Kolcz, A.; Schlaikjer, A.; Gupta, P. Large-scale high-precision topic modeling on twitter. In Proceedings of the 20th ACM SIGKDD international conference on Knowledge discovery and data mining, New York, NY, USA, 24-27 August 2014; ACM: New York, NY, USA, 2014; pp. 1907-1916.

13. Jo, E.S.; Gebru, T. Lessons from archives: Strategies for collecting sociocultural data in machine learning. In Proceedings of the 2020 Conference on Fairness, Accountability, and Transparency, Barcelona, Spain, 27-30 January 2020; pp. 306-316.

14. Zhou, Z.H. A brief introduction to weakly supervised learning. Natl. Sci. Rev. 2017, 5, 44-53. [CrossRef]

15. Helmstetter, S.; Paulheim, H. Weakly Supervised Learning for Fake News Detection on Twitter. In Proceedings of the 2018 IEEE/ACM International Conference on Advances in Social Networks Analysis and Mining (ASONAM), Barcelona, Spain, 28-31 August 2018; IEEE: Piscataway, NJ, USA, 2018; pp. 274-277.

16. Gupta, A.; Kumaraguru, P. Credibility Ranking of Tweets During High Impact Events. In Proceedings of the 1st Workshop on Privacy and Security in Online Social Media, Lyon, France, 17 April 2012; ACM: New York, NY, USA, 2012; PSOSM '12, pp. 2:2-2:8. [CrossRef]

17. Mohd Shariff, S.; Zhang, X.; Sanderson, M. User Perception of Information Credibility of News on Twitter. In Proceedings of the 36th European Conference on IR Research on Advances in Information Retrieval, ECIR 2014, Amsterdam, The Netherlands, 13-16 April 2014; Springer Inc.: New York, NY, USA, 2014; Volume 8416, pp. 513-518. [CrossRef]

18. Sikdar, S.; Adali, S.; Amin, M.; Abdelzaher, T.; Chan, K.; Cho, J.H.; Kang, B.; O’Donovan, J. Finding true and credible information on Twitter. In Proceedings of the 17th International Conference on Information Fusion (FUSION), Salamanca, Spain, 7-10 July 2014; pp. 1-8.

19. Castillo, C.; Mendoza, M.; Poblete, B. Information Credibility on Twitter. In Proceedings of the 20th International Conference on World Wide Web, Hyderabad, India, 28 March-1 April 2011; ACM: New York, NY, USA, 2011; WWW '11, pp. 675-684. [CrossRef] 
20. Ahmed, H.; Traore, I.; Saad, S. Detection of Online Fake News Using N-Gram Analysis and Machine Learning Techniques. In Proceedings of the International Conference on Intelligent, Secure, and Dependable Systems in Distributed and Cloud Environments, Vancouver, BC, Canada, 26-28 October 2017; Springer: Berlin/Heidelberg, Germany, 2017; pp. 127-138.

21. Horne, B.D.; Adali, S. This just in: fake news packs a lot in title, uses simpler, repetitive content in text body, more similar to satire than real news. arXiv 2017, arXiv:1703.09398.

22. Zhao, Z.; Zhao, J.; Sano, Y.; Levy, O.; Takayasu, H.; Takayasu, M.; Li, D.; Havlin, S. Fake news propagate differently from real news even at early stages of spreading. arXiv 2018, arXiv:1803.03443.

23. Vosoughi, S.; Roy, D.; Aral, S. The spread of true and false news online. Science 2018, 359, 1146-1151. [CrossRef] [PubMed]

24. Verstraete, M.; Bambauer, D.E.; Bambauer, J.R. Identifying and countering fake news. In Arizona Legal Studies Discussion Paper; SSRN: Rochester, NY, USA, 2017.

25. Azab, A.E.; Idrees, A.M.; Mahmoud, M.A.; Hefny, H. Fake Account Detection in Twitter Based on Minimum Weighted Feature set. World Acad. Sci. Eng. Technol. Int. J. Comput. Electr. Autom. Control Inf. Eng. 2015, 10, 13-18.

26. Benevenuto, F.; Magno, G.; Rodrigues, T.; Almeida, V. Detecting spammers on twitter. In Proceedings of the Collaboration, Electronic Messaging, Anti-Abuse and Spam Conference (CEAS), Redmond, WA, USA, 13-14 July 2010.

27. Gurajala, S.; White, J.S.; Hudson, B.; Matthews, J.N. Fake Twitter Accounts: Profile Characteristics Obtained Using an Activitybased Pattern Detection Approach. In Proceedings of the 2015 International Conference on Social Media \& Society, Toronto, ON, Canada, 27-29 July 2015; ACM: New York, NY, USA, 2015; SMSociety '15, pp. 9:1-9:7. [CrossRef]

28. Stringhini, G.; Kruegel, C.; Vigna, G. Detecting Spammers on Social Networks. In Proceedings of the 26th Annual Computer Security Applications Conference, Orlando, FL, USA, 4-8 December 2010; ACM: New York, NY, USA, 2010; ACSAC '10, pp. 1-9. [CrossRef]

29. Fellbaum, C. WordNet: An Electronic Lexical Database; MIT Press: Cambridge, MA, USA, 1998.

30. Bird, S.; Loper, E. NLTK: the natural language toolkit. In Proceedings of the ACL 2004 on Interactive poster and demonstration sessions. Association for Computational Linguistics, Barcelona, Spain, 21-26 July 2004; p. 31.

31. Le, Q.; Mikolov, T. Distributed Representations of Sentences and Documents. In 31st International Conference on Machine Learning, Proceedings of the Machine Learning Research, Beijing, China, 21-26 June 2014; Xing, E.P., Jebara, T., Eds.; PMLR: Bejing, China, 2014; Volume 32, pp. 1188-1196.

32. Mikolov, T.; Chen, K.; Corrado, G.; Dean, J. Efficient Estimation of Word Representations in Vector Space. arXiv 2013, arXiv:1301.3781.

33. Blei, D.M.; Ng, A.Y.; Jordan, M.I. Latent Dirichlet Allocation. J. Mach. Learn. Res. 2003, 3, 993-1022.

34. Hoffman, M.; Bach, F.R.; Blei, D.M. Online Learning for Latent Dirichlet Allocation. In Advances in Neural Information Processing Systems 23; Lafferty, J.D., Williams, C.K.I., Shawe-Taylor, J., Zemel, R.S., Culotta, A., Eds.; Curran Associates, Inc.: Red Hook, NY, USA, 2010; pp. 856-864.

35. Teh, Y.W.; Jordan, M.I.; Beal, M.J.; Blei, D.M. Hierarchical Dirichlet Processes. J. Am. Stat. Assoc. 2006, 101, 1566-1581. [CrossRef]

36. Teh, Y.W.; Jordan, M.I. Hierarchical Bayesian Nonparametric Models with Applications. In Bayesian Nonparametrics: Principles and Practice; Hjort, N., Holmes, C., Müller, P., Walker, S., Eds.; Cambridge University Press: Cambridge, UK, 2010.

37. Wang, C.; Paisley, J.W.; Blei, D.M. Online Variational Inference for the Hierarchical Dirichlet Process. In Fourteenth International Conference on Artificial Intelligence and Statistics (AISTATS-11), Ft. Lauderdale, FL, USA, 2011; Gordon, G.J., Dunson, D.B., Eds.; Proceedings of Machine Learning Research: Cambridge, UK, 2011; Volume 15, pp. 752-760.

38. Lesk, M. Automatic Sense Disambiguation Using Machine Readable Dictionaries: How to Tell a Pine Cone from an Ice Cream Cone. In Proceedings of the 5th Annual International Conference on Systems Documentation, Toronto, ON, Canada, 8-11 June 1986; ACM: New York, NY, USA, 1986; SIGDOC '86, pp. 24-26. [CrossRef]

39. Baccianella, S.; Esuli, A.; Sebastiani, F. SentiWordNet 3.0: An Enhanced Lexical Resource for Sentiment Analysis and Opinion Mining. In Proceedings of the Seventh International Conference on Language Resources and Evaluation (LREC'10), Valetta, Malta, 2010; Chair, N.C.C., Choukri, K., Maegaard, B., Mariani, J., Odijk, J., Piperidis, S., Rosner, M., Tapias, D., Eds.; European Language Resources Association (ELRA): Valletta, Malta, 2010.

40. Unicode Inc. Full Emoji Data, v5.0. Available online: http://unicode.org/emoji/charts/full-emoji-list.html (accessed on 20 May 2017).

41. Berry, N. Emoticon Analysis in Twitter. Available online: http:// datagenetics.com/blog/october52012/index.html (accessed on 9 May 2017).

42. De Smedt, T.; Daelemans, W. Pattern for Python. J. Mach. Learn. Res. 2012, 13, 2063-2067.

43. Smedt, T.D.; Daelemans, W. “Vreselijk mooi!" (terribly beautiful): A Subjectivity Lexicon for Dutch Adjectives. In LREC; Calzolari, N., Choukri, K., Declerck, T., Dogan, M.U., Maegaard, B., Mariani, J., Odijk, J., Piperidis, S., Eds.; European Language Resources Association (ELRA): Luxemburg, 2012; pp. 3568-3572.

44. Molina, L.C.; Belanche, L.; Nebot, À. Feature selection algorithms: A survey and experimental evaluation. In Proceedings of the 2002 IEEE International Conference on Data Mining, Maebashi City, Japan, 9-12 December 2002; IEEE: Piscataway, NJ, USA, 2002; pp. 306-313.

45. Hsu, C.W.; Chang, C.C.; and Lin, C.J.; A Practical Guide to Support Vector Classification. 2003. Available online: https: //www.csie.ntu.edu.tw/ cjlin/papers/guide/guide.pdf (accessed on 28 April 2021).

46. Heaton, J. Introduction to Neural Networks for Java, 2nd ed.; Heaton Research, Inc.: St. Louis, MO, USA, 2008. 
47. Bergstra, J.; Bengio, Y. Random search for hyper-parameter optimization. J. Mach. Learn. Res. 2012, 13, $281-305$.

48. Frénay, B.; Verleysen, M. Classification in the presence of label noise: a survey. IEEE Trans. Neural Netw. Learn. Syst. 2014, 25, 845-869. [CrossRef] [PubMed]

49. Lawrence, N.D.; Schölkopf, B. Estimating a kernel Fisher discriminant in the presence of label noise. ICML 2001, 1, 306-313.

50. Natarajan, N.; Dhillon, I.S.; Ravikumar, P.K.; Tewari, A. Learning with noisy labels. Adv. Neural Inf. Process. Syst. 2013, $26,1196-1204$. 